\section{Robust Stability of Time-Varying Uncertain Systems With Rational Dependence on the Uncertainty}

\author{
Graziano Chesi, Senior Member, IEEE
}

\begin{abstract}
Robust stability of time-varying uncertain systems is a key problem in automatic control. This note considers the case of linear systems with rational dependence on an uncertain time-varying vector constrained in a polytope, which is typically addressed in the literature by using the linear fractional representation (LFR). A novel sufficient condition for robust stability is derived in terms of a linear matrix inequality (LMI) feasibility test by exploiting homogeneous polynomial Lyapunov functions, the square matrix representation and an extended version of Polya's theorem which considers structured matrix polynomials. It is shown that this condition is also necessary for second-order systems, and that this condition is less conservative than existing LMI conditions based on the LFR for any order.
\end{abstract}

Index Terms-Linear matrix inequality (LMI), robust stability, square matrix representation (SMR), time-varying uncertainty, uncertain system.

\section{INTRODUCTION}

It is well known that systems with uncertainty is an important area of automatic control. A key problem in this area consists of establishing whether an uncertain linear system is robustly stable, i.e., stable for a set of admissible values of the uncertainty. Various contributions have been proposed in the literature for addressing this problem, mainly based on linear matrix inequalities (LMIs) (see, e.g., [1] and references therein).

One of the first cases that have been considered concerns time-invariant uncertainty that affects affinely the system. In this case, the uncertainty is typically assumed to belong to a polytope, and numerous conditions have been proposed by exploiting, firstly, common quadratic Lyapunov functions, and successively, parameter-dependent quadratic Lyapunov functions in order to reduce the conservatism. Some of these conditions also allow one to consider different dependence on the uncertainty, e.g., bilinear, polynomial, and rational (see, e.g., [2]-[8]).

Another important case concerns time-varying uncertainty. Also in this case, the pioneering methods considered systems with affine dependence on uncertainty constrained in a polytope, and provided conditions based on common quadratic Lyapunov functions. In order to cope with the conservatism of these conditions, the use of non-quadratic common Lyapunov functions was proposed, e.g., piecewise quadratic, polynomial, and polyhedral. Some of these conditions also allow one to consider different dependence on the uncertainty and the presence of bounds on the variation rate of the uncertainty (see, e.g., [2], [8]-[16]).

This note considers uncertain linear systems with rational dependence on time-varying uncertainty constrained in a polytope, which is typically addressed in the literature by using the linear fractional representation (LFR). A sufficient condition for robust stability of the uncertain system is proposed in terms of an LMI feasibility test by exploiting

Manuscript received September 24, 2009; revised December 12, 2009; accepted May 18, 2010. Date of publication June 21, 2010; date of current version October 06, 2010. This work was supported in part by the Research Grants Council of Hong Kong (Grant HKU711208E). Recommended by Associate Editor D. Arzelier.

The author is with the Department of Electrical and Electronic Engineering, University of Hong Kong, Hong Kong, China (e-mail: chesi@eee.hku.hk).

Digital Object Identifier 10.1109/TAC.2010.2053470 homogeneous polynomial Lyapunov functions ${ }^{1}$, the square matrix representation (SMR), and an extended version of Polya's theorem which considers structured matrix polynomials. It is shown that this condition is also necessary for second-order systems, and that this condition is less conservative than existing LMI conditions based on the LFR for any order.

\section{PRELiminaries}

\section{A. Problem Formulation}

The notation used throughout the note is as follows: $\mathbb{N}, \mathbb{R}$ : natural and real number sets; $0_{n}$ : origin of $\mathbb{R}^{n} ; \mathbb{R}_{0}^{n}: \mathbb{R}^{n} \backslash\left\{0_{n}\right\} ; I_{n}: n \times n$ identity matrix; $A^{\prime}$ : transpose of $A ; A>0(A \geq 0)$ : symmetric positive definite (semidefinite) matrix $A$; he $(A)=A+A^{\prime} ; \nabla v(x)$ : first derivative row vector of the function $v(x) ; \operatorname{conv}\{a, b, \ldots\}$ : convex hull of vectors $a, b, \ldots$.

Let us consider the uncertain system

$$
\left\{\begin{array}{l}
\dot{x}(t)=A(p(t)) x(t) \\
p(t) \in \mathcal{P}
\end{array}\right.
$$

where $x(t) \in \mathbb{R}^{n}$ is the state, $p(t) \in \mathbb{R}^{q}$ is the time-varying uncertain vector, $A: \mathbb{R}^{q} \rightarrow \mathbb{R}^{n \times n}$ is a matrix rational function, and $\mathcal{P} \subset \mathbb{R}^{q}$ is a given bounded convex polytope. The matrix rational function $A(p(t))$ is expressed as

$$
A(p(t))=\frac{B(p(t))}{b(p(t))}
$$

where $B: \mathbb{R}^{q} \rightarrow \mathbb{R}^{n \times n}$ and $b: \mathbb{R}^{q} \rightarrow \mathbb{R}$ are polynomials. We denote the degree of $B(p(t))$ in $p(t)$ with $\delta$.

Throughout the note, we assume that:

- $p(t)$ ensures the existence of the solution $x(t)$ of $(1)$;

- the polynomial $b(p(t))$ satisfies

$$
b(p(t))>0 \quad \forall p(t) \in \mathcal{P}
$$

which is equivalent to say that the LFR of (1) is well-posed [11] ${ }^{2}$. The problem considered in this note is to establish whether the origin is an asymptotically stable equilibrium point for the system (1).

\section{B. Square Matrix Representation}

Before proceeding, we briefly introduce a key tool that will be exploited in the next sections to derive the proposed conditions. For $x \in$ $\mathbb{R}^{n}$, let $h(x)$ be a homogeneous polynomial, i.e., a polynomial with all monomials of the same degree, and let $2 \mathrm{~m}$ be the degree of $h(x)$. Let $x^{\{m\}} \in \mathbb{R}^{\sigma(n, m)}$ be a vector containing all monomials of degree less equal to $m$ in $x$, where $\sigma(n, m)$ is the number of such monomials given by

$$
\sigma(n, m)=\frac{(n+m-1) !}{(n-1) ! m !}
$$

Then, $h(x)$ can be expressed via the SMR introduced in [17] as

$$
h(x)=x^{\{m\}^{\prime}}(H+L(\alpha)) x^{\{m\}}
$$

${ }^{1}$ These functions have been exploited by several authors, in particular in [15] they are used with polynomial dependence on the uncertainty for the case of bounds on the variation rate of the uncertainty.

${ }^{2}$ This because $b(p)=\operatorname{det}(I-D E(p))$, where $D$ and $E(p)$ are two of the five matrices see, e.g., [13], of the LFR of (1), and the LFR is well-posed if $b(p) \neq 0$ for all $p \in \mathcal{P}$. 
where $H=H^{\prime} \in \mathbb{R}^{\sigma(n, m) \times \sigma(n, m)}$ is a symmetric matrix such that $h(x)=x^{\{m\}^{\prime}} H x^{\{m\}}, L(\alpha)=L(\alpha)^{\prime} \in \mathbb{R}^{\sigma(n, m) \times \sigma(n, m)}$ is a linear parametrization of the set

$$
\mathcal{L}_{n, m}=\left\{L=L^{\prime}: x^{\{m\}^{\prime}} L x^{\{m\}}=0\right\}
$$

and $\alpha \in \mathbb{R}^{\omega(n, m)}$ is a vector of free parameters, where $\omega(n, m)$ is the dimension of the linear subspace $\mathcal{L}_{n, m}$ given by

$$
\omega(n, m)=\frac{1}{2} \sigma(n, m)(\sigma(n, m)+1)-\sigma(n, 2 m) .
$$

The matrices $H$ and $H+L(\alpha)$ are referred to as SMR matrix and complete SMR matrix, respectively, of $h(x)$.

The SMR is useful because it allows one to investigate positivity of polynomials. Indeed, one can establish whether $h(x)$ is a sum of squares of polynomials (SOS) by solving a convex optimization problem with linear matrix inequalities (LMIs) [17]. Specifically, $h(x)$ is SOS if and only if there exists $\alpha$ such that

$$
H+L(\alpha) \geq 0
$$

which is an LMI feasibility test since $H$ is constant and $L(\alpha)$ is a linear function. LMI feasibility tests can be checked by solving a convex optimization problem, see for instance [2] (see also [8] for details and algorithms).

\section{Robust StABILITy CONDITION}

First of all, we can rewrite (1) as

$$
\left\{\begin{array}{l}
\dot{x}(t)=D(s(t)) x(t) \\
s(t) \in \mathcal{S}
\end{array}\right.
$$

where $s=\left(s_{1}, \ldots, s_{r}\right)^{\prime} \in \mathbb{R}^{r}$ is related to $p$ by

$$
p=\sum_{i=1}^{r} s_{i} p^{(i)}
$$

and $p^{(1)}, \ldots, p^{(r)} \in \mathbb{R}^{q}$ are the vertices of $\mathcal{P}, \mathcal{S}$ is the simplex

$$
\mathcal{S}=\left\{s \in \mathbb{R}^{r}: \sum_{i=1}^{r} s_{i}=1, s_{i} \geq 0\right\}
$$

and $D: \mathbb{R}^{r} \rightarrow \mathbb{R}^{n \times n}$ is a function that we express as

$$
D(s)=\frac{C(s)}{c(s)}
$$

where $C: \mathbb{R}^{r} \rightarrow \mathbb{R}^{n \times n}$ and $c: \mathbb{R}^{r} \rightarrow \mathbb{R}$ are homogeneous polynomials such that $C(s)=B(p)$ and $c(s)=b(p)$. The degree of $C(s)$ is hence $\delta$.

Next, we look for a continuous function $v: \mathbb{R}^{n} \rightarrow \mathbb{R}$ such that

$$
\left\{\begin{array}{l}
v(x)>0 \forall x \in \mathbb{R}_{0}^{n} \text { and } 0=v(0) \\
\dot{v}(x, s)<0 \forall x \in \mathbb{R}_{0}^{n} \forall s \in \mathcal{S}
\end{array}\right.
$$

where $\dot{v}(x, s)$ is the time derivative of $v(x)$ along the trajectories of the system (9) given by $\dot{v}(x, s)=\nabla v(x)^{\prime} D(s) x$, being $\nabla v(x) \in \mathbb{R}^{n}$ the gradient of $v(x)$. To this end, we consider Lyapunov functions that are homogeneous polynomials in $x$. Hence, the generic candidate Lyapunov function can be written by using the SMR introduced in Section II-B as

$$
v(x)=x^{\{m\}^{\prime}} V x^{\{m\}}
$$

where $m \in \mathbb{N}$ defines the degree of $v(x)$, equal to $2 m$, and $V=V^{\prime} \in$ $\mathbb{R}^{\sigma(n, m) \times \sigma(n, m)}$ is a symmetric matrix. One has that $\dot{v}(x, s)$ is given by

$$
\dot{v}(x, s)=\text { he }\left(x^{\{m\}^{\prime}} V E(x, s)\right)
$$

where

$$
E(x, s)=\left.\frac{d x^{\{m\}}}{d t}\right|_{\dot{x}=D(s) x} .
$$

The vector function $E(x, s)$ can be rewritten as

$$
E(x, s)=\frac{1}{c(s)} \frac{d x^{\{m\}}}{d x} C(s) x=\frac{F(s)}{c(s)} x^{\{m\}}
$$

where $F: \mathbb{R}^{r} \rightarrow \mathbb{R}^{\sigma(n, m) \times \sigma(n, m)}$ is a homogeneous polynomial of degree $\delta$. The matrix $F(s)$ can be calculated either via a simple algorithm or via the formula

$$
F(s)=\left(K^{\prime} K\right)^{-1} K^{\prime}\left(\sum_{i=0}^{m-1} I_{n m-1-i} \otimes C(s) \otimes I_{n^{i}}\right) K
$$

where $K \in \mathbb{R}^{n^{m}} \times \sigma(n, m)$ is the matrix satisfying

$$
x^{[m]}=K x^{\{m\}}
$$

and $x^{[m]}$ denotes the $m$-th Kronecker power of $x$. Therefore, the time derivative of $v(x)$ can be rewritten as

$$
\dot{v}(x, s)=x^{\{m\}^{\prime}} \text { he }\left(\frac{V F(s)}{c(s)}\right) x^{\{m\}}
$$

and the Lyapunov inequalities in (13) become

$$
\left\{\begin{array}{l}
x^{\{m\}^{\prime}} V x^{\{m\}}>0 \quad \forall x \in \mathbb{R}_{0}^{n} \\
x^{\{m\}^{\prime}} \text { he }(V F(s)) x^{\{m\}}<0 \quad \forall x \in \mathbb{R}_{0}^{n} \forall s \in \mathcal{S} .
\end{array} .\right.
$$

At this point, let us observe that (21) holds if and only if

$$
\left\{\begin{array}{l}
0<x^{\{m\}^{\prime}} V x^{\{m\}} \quad \forall x \in \mathbb{R}_{0}^{n} \\
0>x^{\{m\}^{\prime}} \text { he }(V G(s, k)) x^{\{m\}} \quad \forall x \in \mathbb{R}_{0}^{n} \forall s \in \mathcal{S}
\end{array}\right.
$$

where $k \geq 0$ is an integer related to Polya's theorem, and

$$
G(s, k)=\left(\sum_{i=1}^{r} s_{i}\right)^{k} F(s) .
$$

Indeed, one has

$$
G(s, k)=F(s) \quad \forall s \in \mathcal{S} \forall k .
$$

As second step, let us express $G(s, k)$ as

$$
G(s, k)=\left(G_{1}, \ldots, G_{l}\right)\left(s^{\{\delta+k\}} \otimes I_{\sigma(n, m)}\right)
$$

where $G_{1}, \ldots, G_{l} \in \mathbb{R}^{\sigma(n, m) \times \sigma(n, m)}$ and $l=\sigma(r, \delta+k)$.

Theorem 1: Let $m \geq 1$ and $k \geq 0$ be integers, and let $L: \mathbb{R}^{\omega(n, m)} \rightarrow \mathbb{R}^{\sigma(n, m) \times \sigma(n, m)}$ be a linear parametrization of the set $\mathcal{L}_{n, m}$ in (6). The origin of (1) is asymptotically stable if there exist variables $V=V^{\prime} \in \mathbb{R}^{\sigma(n, m) \times \sigma(n, m)}$ and $\alpha^{(1)}, \ldots, \alpha^{(l)} \in \mathbb{R}^{\omega(n, m)}$ such that the following system of LMIs holds:

$$
\left\{\begin{array}{l}
V>0 \\
\operatorname{he}\left(V G_{i}\right)+L\left(\alpha^{(i)}\right)<0 \forall i=1, \ldots, l .
\end{array}\right.
$$


Proof: Let us suppose that (26) holds for some $V=V^{\prime}$ and $\alpha^{(1)}, \ldots, \alpha^{(l)}$. Let us pre-multiply and post-multiply the LMIs in (26) by $x^{\{m\}^{\prime}}$ and $x^{\{m\}}$, respectively. Since $x^{\{m\}} \neq 0_{\sigma(n, m)} \quad \forall x \in \mathbb{R}_{0}^{n}$, we get from the first LMI that $v(x)>0$ for all $x \in \mathbb{R}_{0}^{n}$ where $v(x)$ is as in (14). Similarly, from the second LMI in (26), we get

$$
0>x^{\{m\}^{\prime}}\left(\operatorname{he}\left(V G_{i}\right)+L\left(\alpha^{(i)}\right)\right) x^{\{m\}}=x^{\{m\}^{\prime}} \text { he }\left(V G_{i}\right) x^{\{m\}}
$$

for all $x \in \mathbb{R}_{0}^{n}$ and for all $i=1, \ldots, l$. Let us define

$$
h(x, s)=\left(h_{1}(x), \ldots, h_{l}(x)\right)^{\prime} s^{\{\delta+k\}}
$$

where $h_{i}(x)=x^{\{m\}^{\prime}}$ he $\left(V G_{i}\right) x^{\{m\}}$. It follows that

$$
0>h(x, s) \quad \forall x \in \mathbb{R}_{0}^{n} \forall s \in \mathcal{S}
$$

since $h_{i}(x)$ is negative definite for all $i=1, \ldots, l$, and since the entries $z_{1}(s), \ldots, z_{l}(s)$ of $s^{\{\delta+k\}}$ satisfy

$$
\left\{\begin{array}{l}
z_{i}(s) \geq 0 \quad \forall s \in \mathcal{S} \\
\forall s \in \mathcal{S} \exists i \in[1, l]: z_{i}(s)>0 .
\end{array}\right.
$$

Lastly, one has that $h(x, s)=x^{\{m\}^{\prime}}$ he $(V G(s, k)) x^{\{m\}}$ and hence (22) holds.

Theorem 1 provides a sufficient condition for establishing robust asymptotical stability of the origin of the system (1) via an LMI feasibility test. The number of scalar variables in this condition is equal to

$$
\tau=\frac{1}{2} \sigma(n, m)(\sigma(n, m)+1)+l \omega(n, m) .
$$

Theorem 2: Let us suppose that the origin of (1) is asymptotically stable and $n=2$. Then, the condition of Theorem 1 is sufficient and necessary, i.e.,

$$
\exists m, k:(26) \text { holds for some } V=V^{\prime} \text { and } \alpha^{(1)}, \ldots, \alpha^{(l)} \text {. }
$$

Proof: Let the origin of (1) be asymptotically stable, and let $v(x)$ be a Lyapunov function satisfying (13) (this Lyapunov function can be chosen polynomial [18]). Let us consider $n=2$. In this case there is no gap between positive polynomials and SOS polynomials, see for instance [19] and references therein. In particular, the homogeneous polynomial $v(x)$ of degree $2 m$ is positive definite if and only if there exists $V=V^{\prime} \in \mathbb{R}^{\sigma(n, m) \times \sigma(n, m)}$ such that [8], [12]

$$
\left\{\begin{array}{l}
v(x)=x^{\{m\}^{\prime}} V x^{\{m\}} \\
V>0
\end{array} .\right.
$$

Similarly, one has that

$$
\left\{\begin{array}{l}
\dot{v}(x, s)=x^{\{m\}^{\prime}} U(s) x^{\{m\}} \\
U(s)<0 \forall s \in \mathcal{S}
\end{array}\right.
$$

From (20), one has that $U(s)$ must satisfy

$$
x^{\{m\}^{\prime}} U(s) x^{\{m\}}=x^{\{m\}^{\prime}} \text { he }\left(\frac{V F(s)}{c(s)}\right) x^{\{m\}} .
$$

This condition holds if and only if $U(s)$ can be written as

$$
U(s)=\frac{h \mathrm{e}(V F(s))+L(\phi(s))}{c(s)}
$$

for some function $\phi: \mathbb{R}^{r} \rightarrow \mathbb{R}^{\omega(n, m)}$, where $L(\cdot)$ is a linear parametrization of the set $\mathcal{L}_{n, m}$ in (6). Since $\mathcal{S}$ is compact, $L(\cdot)$ continuous, and $c(s)>0$ for all $s \in \mathcal{S}$, it follows that there exists a polynomial $\theta: \mathbb{R}^{r} \rightarrow \mathbb{R}^{\omega(n, m)}$ such that

$$
\operatorname{he}(V F(s))+L(\theta(s))<0 \quad \forall s \in \mathcal{S} \text {. }
$$

Let $a_{1}$ be the degree of $\theta(s)$, and let us define

$$
T(s)=\left(\sum_{i=1}^{r} s_{i}\right)^{a_{2}} \operatorname{he}(V F(s))+\left(\sum_{i=1}^{r} s_{i}\right)^{a_{3}} L(\theta(s))
$$

where $a_{2}=\max \left\{0, a_{1}-\delta\right\}$ and $a_{3}=\max \left\{0, \delta-a_{1}\right\}$. Since $L(\cdot)$ is a matrix linear function, it follows that $T(s)$ is a homogeneous polynomial of degree $a_{4}=\max \left\{\delta, a_{1}\right\}$.

Next, from [20], one has that $T(s)<0$ for all $s \in \mathcal{S}$ if and only if there exists an integer $a_{5} \geq 0$ such that the homogeneous polynomial

$$
R(s)=\left(\sum_{i=1}^{r} s_{i}\right)^{a_{5}} T(s)
$$

has negative definite matrix coefficients. Let us define $k=a_{5}+a_{4}-\delta$. We have that $R(s)$ can be expressed as

$$
R(s)=\left(R_{1}, \ldots, R_{l}\right)\left(s^{\{\delta+k\}} \otimes I_{\sigma(n, m)}\right)
$$

where $R_{1}, \ldots, R_{l}$ are the matrix coefficients of $R(s)$. The $i$-th matrix coefficient is given by

$$
R_{i}=\operatorname{he}\left(V G_{i}\right)+L\left(\alpha^{(i)}\right)
$$

where $G_{i}$ is defined as in (25), and $\alpha^{(i)}$ is a suitable vector. Therefore, $R_{i}<0$ for all $i=1, \ldots, l$, and hence (26) holds for the constructed $V$ and $\alpha^{(1)}, \ldots, \alpha^{(l)}$.

Remark 1: Theorem 2 states that the stability condition of Theorem 1 is not only sufficient but also necessary in the case of second-order systems for any degree of the dependence on the uncertainty. It should be remarked that existing LMI conditions based on the LFR are only sufficient for these systems.

Remark 2: The condition provided in Theorem 1 contains an extended version of Polya's theorem, which investigates positive definiteness of structured matrix polynomials. This is clarified in the following result.

Corollary 1: Let $Q=Q^{\prime}: \mathbb{R}^{r} \rightarrow \mathbb{R}^{\sigma(n, m) \times \sigma(n, m)}$ be a homogeneous polynomial of degree $\delta$, and let $L: \mathbb{R}^{\omega(n, m)} \rightarrow \mathbb{R}^{\sigma(n, m) \times \sigma(n, m)}$ be a linear parametrization of the set $\mathcal{L}_{n, m}$ in (6). Then,

$$
\exists \phi(s) \text { continuous : } Q(s)+L(\phi(s))>0 \quad \forall s \in \mathcal{S}
$$

if and only if

$$
\exists k, \alpha^{(1)}, \ldots, \alpha^{(l)}: Z_{i}+L\left(\alpha^{(i)}\right)>0 \quad \forall i=1, \ldots, l
$$

where $Z_{i}=Z_{i}^{\prime} \in \mathbb{R}^{\sigma(n, m) \times \sigma(n, m)}, i=1 \ldots, l$, satisfy

$$
\left(\sum_{i=1}^{r} s_{i}\right)^{k} Q(s)=\left(Z_{1}, \ldots, Z_{l}\right)\left(s^{\{\delta+k\}} \otimes I_{\sigma(n, m)}\right) .
$$


Let us observe that the extended version of Polya's theorem provided in Corollary 1 contains the original Polya's theorem for $m=1$ (in this case $\sigma(n, m)=n$ and $\left.L(\cdot)=0_{n \times n}\right)$.

Theorem 3: Let us suppose that the origin of (1) is asymptotically stable, and that the degree $\delta$ of $F(s)$ is 1 . Then, (26) holds with some $k \geq 0$ if and only if it holds with $k=0$.

Proof: Since $\delta=1$, one has that $x^{\{m\}^{\prime}}$ he $(V F(s)) x^{\{m\}}$ is a linear function of $s$, which is negative for all $x \in \mathbb{R}_{0}^{n}$ and $s \in \mathcal{S}$ if and only if

$$
f_{i}(x)<0 \forall x \in \mathbb{R}_{0}^{n} \forall i=1, \ldots, r
$$

where $f_{i}(x)$ is $x^{\{m\}^{\prime}}$ he $(V F(s)) x^{\{m\}}$ evaluated at the $i$-th vertex of $\mathcal{S}$. Each $f_{i}(x)$ can be written as

$$
f_{i}(x)=x^{\{m\}^{\prime}}\left(\operatorname{he}\left(V G_{i}\right)+L\left(\alpha^{(i)}\right)\right) x^{\{m\}}
$$

where $G_{i}$ is defined as in (25) for $k=0$, and $\alpha^{(i)}$ is a suitable vector. Therefore, the condition $f_{i}(x)<0$ for all $x \in \mathbb{R}_{0}^{n}$ and $i=1, \ldots, r$ coincides with (26).

Theorem 3 explains that, in the case of systems with linear dependence on the time-varying uncertainty, one can select $k=0$ without introducing conservatism with respect to cases with $k>0$. It is useful to observe that, for $\delta=1$ and $k=0$, the condition of Theorem 1 boils down to the condition provided in [8], [12].

Theorem 4: Let us suppose that the LFR conditions of Corollaries 1 and 2 in [13] are satisfied for some $m$. Then, there exists $k$ such that (26) holds for the same $m$.

Proof: The LFR conditions of [13, Coroll. 1 and 2] are the LMIs

$$
\left\{\begin{array}{l}
P_{m}>0 \\
M_{m}^{(i)}<0 \forall i=1, \ldots, r
\end{array}\right.
$$

where $P_{m}$ is the matrix $V$ in (14) and $M_{m}^{(i)}=$ $R_{m}\left(P_{m}, G_{m}, H_{m}, E_{i}\right)+N_{m}\left(\beta_{i}\right)$ for Corollary 1 , or $M_{m}^{(i)}=R_{m}\left(P_{m}, G_{m, i}, H_{m, i}, E_{i}\right)+N_{m}\left(\beta_{i}\right)$ for Corollary 2, provides the derivative of $v(x)$ at the $i$-th vertex of $\mathcal{P}$ according to

$$
\begin{aligned}
\left.\dot{v}(x)\right|_{p=p^{(i)}} & =y(x ; \varrho)^{\prime} M_{m}^{(i)} y(x ; \varrho) \\
y(x ; \varrho) & =\left(\begin{array}{c}
x^{\{m\}} \\
\varrho \otimes x^{\{m-1\}}
\end{array}\right)
\end{aligned}
$$

being $\varrho$ the output of the LFR of (1) (see [13] for further details). Hence, one has that $\dot{v}(x, s)=y(x ; \varrho)^{\prime} M(s) y(x ; \varrho)$ where $M(s)=$ $\sum_{i=1}^{r} s_{i} M_{m}^{(i)}$. Moreover, from the LFR of (1) it follows that $\varrho=$ $(I-D \tilde{E}(s))^{-1} C x$ where $\tilde{E}(s)=\sum_{i=1}^{r} s_{i} E_{i}$, and hence

$$
y(x ; \varrho)=\frac{Y(s)}{d(s)} x^{\{m\}}, d(s)=\operatorname{det}(I-D \tilde{E}(s))
$$

for some matrix polynomial $Y(s)$. This implies that

$$
\dot{v}(x, s)=x^{\{m\}^{\prime}} U(s) x^{\{m\}}, U(s)=\frac{Y(s)^{\prime} M(s) Y(s)}{d(s)^{2}} .
$$

If the LFR conditions of Corollaries 1 and 2 in [13] are satisfied, then one has $M(s)<0$ for all $s \in \mathcal{S}$, moreover $d(s) \neq 0$ for all $s \in \mathcal{S}$ since the LFR is well-posed, and $Y(s)$ has full column rank. Consequently, $U(s)<0$ for all $s \in \mathcal{S}$, and the proof proceeds now as the proof of Theorem 2 from the point "From (20), one has that $U(s)$ must satisfylldots" which shows the existence of $k$ such that (26) holds.

Theorem 4 states that the condition of Theorem 1 is satisfied whenever the LFR conditions in [13] (which also contain the LFR conditions in [11]) are satisfied.
TABLE I

LOWER BOUNDS $\hat{\zeta}$ (PROVIDED BY THEOREM 1 FOR $k=0$ ) AND $\hat{\zeta}_{L F R}$ (PROVIDED BY THE LFR CONDITION IN [13]) OF $\zeta^{*}$. THE QUANTITIES $\tau$ AND $\tau_{L F R}$ ARE THE NUMBER OF SCALAR VARIABLES OF THE TWO LMI CONDITIONS

\begin{tabular}{c|cccc}
$m$ & $\hat{\zeta}$ & $\tau$ & $\hat{\zeta}_{L F R}$ & $\tau_{L F R}$ \\
\hline 1 & 5.059 & 11 & 4.380 & 31 \\
2 & 9.157 & 176 & 8.670 & 613 \\
3 & 11.353 & 967 & 10.726 & 4149
\end{tabular}

\section{ILLUSTRATIVE EXAMPLE}

Let us consider the uncertain system

$$
\left\{\begin{array}{l}
\dot{x}(t)=A(p(t)) x(t) \\
p(t) \in \mathcal{P}=\operatorname{conv}\left\{(0,0)^{\prime},(\zeta, 0)^{\prime},(0, \zeta)^{\prime}\right\}
\end{array}\right.
$$

where $\zeta \in \mathbb{R}$ defines the size of the polytope of uncertainties and

$$
A(p)=\frac{A_{0}+A_{1} p_{1}+A_{2} p_{2}+A_{3} p_{1}^{2}+A_{4} p_{1} p_{2}}{p_{1}+1}
$$

with

$$
\begin{aligned}
A 0 & =\left(\begin{array}{cccc}
-0.979 & 0.182 & -1.221 & -0.803 \\
0.392 & -0.754 & 1.963 & 0.233 \\
0.013 & -1.185 & -0.795 & 0.735 \\
-0.366 & -0.412 & 0.150 & -1.385
\end{array}\right) \\
A 1 & =\left(\begin{array}{cccc}
-1.801 & 0.489 & -0.845 & -0.952 \\
0.778 & -0.859 & 2.214 & 0.457 \\
-1.837 & -0.567 & -0.732 & 0.119 \\
-1.778 & 0.059 & 0.180 & -1.861
\end{array}\right) \\
A 2 & =\left(\begin{array}{cccc}
-0.334 & 0.041 & 0.607 & -1.287 \\
0.059 & -0.007 & -0.107 & 0.228 \\
0.607 & -0.075 & -1.105 & 2.343 \\
0.026 & -0.003 & -0.047 & 0.099
\end{array}\right) \\
A 3 & =\left(\begin{array}{cccc}
0.066 & -0.042 & -0.217 & -0.055 \\
-0.195 & 0.124 & 0.640 & 0.163 \\
0.448 & -0.285 & -1.472 & -0.375 \\
0.349 & -0.222 & -1.146 & -0.292
\end{array}\right) \\
A 4 & =\left(\begin{array}{cccc}
-1.190 & 0.378 & 1.179 & -1.377 \\
0.211 & -0.067 & -0.209 & 0.244 \\
2.166 & -0.688 & -2.146 & 2.506 \\
0.092 & -0.029 & -0.091 & 0.106
\end{array}\right) .
\end{aligned}
$$

The problem consists of determining the maximum $\zeta$, denoted by $\zeta^{*}$, for which the origin is asymptotically stable (by using the technique in [5], [8] one can verify that $\zeta^{*}=\infty$ for time-invariant uncertainty).

Table I shows the lower bounds $\hat{\zeta}$ and $\hat{\zeta}_{L F R}$ of $\zeta^{*}$ provided respectively by Theorem 1 for $k=0$ and by the LFR condition in [13] $]^{3}$. As we can see, Theorem 1 provides less conservative lower bounds. It is also worth observing that the computational burden of Theorem 1 is smaller (though this may not be always the case).

\section{CONCLUSION}

This note has considered the problem of establishing robust stability of uncertain linear systems with rational dependence on an uncertain time-varying vector constrained in a polytope. A novel sufficient condition has been proposed in terms of a convex optimization problem by exploiting homogeneous polynomial Lyapunov functions, the square matrix representation and an extended version of Polya's theorem which considers structured matrix polynomials. It has been

${ }^{3}$ The matrices $A, B, C, D, E$ in the LFR of this system have size $4 \times 4$, $4 \times 3,3 \times 4,3 \times 3$ and $3 \times 3$. The LFR degree is 2 . 
shown that this condition is also necessary for second-order systems, and that this condition is less conservative than existing LMI conditions based on the LFR for any order.

\section{ACKNOWLEDGMENT}

The author thanks the Associate Editor and the Reviewers for their useful comments.

\section{REFERENCES}

[1] G. Chesi, "LMI techniques for optimization over polynomials in control: A survey," IEEE Trans. Autom. Control, 2010, to be published.

[2] S. Boyd, L. El Ghaoui, E. Feron, and V. Balakrishnan, Linear Matrix Inequalities in System and Control Theory. Philadelphia, PA: SIAM, 1994.

[3] A. Trofino, "Parameter dependent Lyapunov functions for a class of uncertain linear systems: A LMI approach," in Proc. IEEE Conf. Decision and Control, Phoenix, AZ, 1999, pp. 2341-2346.

[4] P.-A. Bliman, "A convex approach to robust stability for linear systems with uncertain scalar parameters," SIAM J. Control Optimiz., vol. 42, no. 6, pp. 2016-2042, 2004.

[5] G. Chesi, A. Garulli, A. Tesi, and A. Vicino, "Polynomially parameter-dependent Lyapunov functions for robust stability of polytopic systems: An LMI approach," IEEE Trans. Autom. Control, vol. 50, no. 3, pp. 365-370, Mar. 2005.

[6] C. W. Scherer, "LMI relaxations in robust control," Eur. J. Control, vol. 12, no. 1, pp. 3-29, 2006.

[7] R. C. L. F. Oliveira and P. L. D. Peres, "Parameter-dependent LMIs in robust analysis: Characterization of homogeneous polynomially parameter-dependent solutions via LMI relaxations," IEEE Trans. Autom. Control, vol. 52, no. 7, pp. 1334-1340, Jul. 2007.

[8] G. Chesi, A. Garulli, A. Tesi, and A. Vicino, Homogeneous Polynomial Forms for Robustness Analysis of Uncertain Systems. New York: Springer, 2009.

[9] F. Blanchini, "Nonquadratic Lyapunov functions for robust control," Automatica, vol. 31, pp. 451-461, 1995.

[10] H. L. S. Almeida, A. Bhaya, D. M. Falcao, and E. Kaszkurewicz, "A team algorithm for robust stability analysis and control design of uncertain time-varying linear systems using piecewise quadratic Lyapunov functions," Int. J. Robust Nonlin. Control, vol. 11, pp. 357-371, 2001.

[11] F. Wang and V. Balakrishnan, "Improved stability analysis and gain-scheduled controller synthesis for parameter-dependent systems," IEEE Trans. Autom. Control, vol. 47, no. 5, pp. 720-734, May 2002.

[12] G. Chesi, A. Garulli, A. Tesi, and A. Vicino, "Homogeneous Lyapunov functions for systems with structured uncertainties," Automatica, vol. 39, no. 6, pp. 1027-1035, 2003.

[13] G. Chesi, A. Garulli, A. Tesi, and A. Vicino, "Robust analysis of LFR systems through homogeneous polynomial Lyapunov functions," IEEE Trans. Autom. Control, vol. 49, no. 7, pp. 1211-1216, Jul. 2004.

[14] J. C. Geromel and P. Colaneri, "Robust stability of time varying polytopic systems," Syst. Control Lett., vol. 55, no. 1, pp. 81-85, 2006.

[15] G. Chesi, A. Garulli, A. Tesi, and A. Vicino, "Robust stability of time-varying polytopic systems via parameter-dependent homogeneous Lyapunov functions," Automatica, vol. 43, no. 2, pp. 309-316, 2007.

[16] F. Blanchini and S. Miani, Set-Theoretic Methods in Control. New York: Birkhauser, 2008.

[17] G. Chesi, A. Tesi, A. Vicino, and R. Genesio, "On convexification of some minimum distance problems," in Proc. European Control Conf., Karlsruhe, Germany, 1999.

[18] F. Blanchini and S. Miani, "A universal class of smooth functions for robust control," IEEE Trans. Autom. Control, vol. 44, no. 3, pp. 641-647, Mar. 1999.

[19] G. Chesi, "On the gap between positive polynomials and SOS of polynomials," IEEE Trans. Autom. Control, vol. 52, no. 6, pp. 1066-1072, Jun. 2007.

[20] C. W. Scherer, "Relaxations for robust linear matrix inequality problems with verifications for exactness," SIAM J. Matrix Anal. Appl., vol. 27, no. 1, pp. 365-395, 2005.

\section{Adaptive Control for Uncertain Continuous-Time Systems Using Implicit Inversion of Prandtl-Ishlinskii Hysteresis Representation}

\author{
Xinkai Chen, Senior Member, IEEE, Takeshi Hisayama, and \\ Chun-Yi Su, Senior Member, IEEE
}

\begin{abstract}
In this note, an implicit inversion approach is introduced to avoid difficulties associated with stability analysis in the direct application of inversion for operator-based hysteresis models. Based on this implicit inversion, an adaptive control algorithm is formulated for continuous-time linear dynamical systems preceded with hysteresis nonlinearities described by the Prandtl-Ishlinskii model. A stability analysis of the controlled system is performed to show that zero-output tracking error can be achieved. Simulation results show the effectiveness of the proposed algorithm.
\end{abstract}

Index Terms-Adaptive control, continuous-time systems, hysteresis, Prandtl-Ishlinskii model.

\section{INTRODUCTION}

Recently, the problem of controlling dynamic systems preceded by unknown hysteresis has received considerable attention. The most common control approach is to construct an inverse hysteresis model to compensate for the effect of the hysteresis [11]. Some recent progress can be referred to, for instance, [4], [6], [7], [13] and the references therein. Essentially, the inversion problem depends on the hysteresis modeling methods. Hysteretic nonlinearities are generally very complicated with multi-values and non-smooth features, such as those in piezo-electric actuators and magnetostrictive actuators, where the operator-based hysteresis models are usually applied [3], [4], [7], [8], [13]. In such cases, the analytic inversion of the hysteresis models is very complicated and remains a challenging task [4], [7]. The main problem is that hysteresis cancellation by direct inversion will introduce compensation errors which, serving as the input signal, may cause difficulties in stability analysis for the closed-loop system.

Various approaches have been proposed in the literature, to avoid difficulties associated with stability analysis, for closed-loop systems that directly use inverse construction for operator-based hysteresis models. One approach is to directly develop the control algorithms without the inverse model construction [10], which is very useful when the hysteresis is represented by differential equations where the inversion is either impossible or extremely difficult [9], [16]. However, this control algorithm may lead to large control input magnitudes. Another approach, instead of directly constructing the inversion from the hysteresis model, is an approximate implicit inversion. This method, associated with the

Manuscript received November 26, 2008; revised February 24, 2010; accepted May 12, 2010. Date of publication June 21, 2010; date of current version October 06, 2010. This work was supported in part by the National Fundamental Research Program of China under Grant 61020106003 and 61074097, and by the 111 Project under Grant B08015. Recommended by Associate Editor J.-F Zhang.

$\mathrm{X}$. Chen is with the Department of Electronic and Information Systems, Shibaura Institute of Technology, Saitama 337-8570, Japan (e-mail: chen@shibaura-it.ac.jp).

T. Hisayama is with the IHI Scube Co., Ltd., Tokyo 104-0028, Japan (e-mail: tawara4946@yahoo.co.jp).

C.-Y. Su is with the Department of Mechanical and Industrial Engineering, Concordia University, Montreal, QC H3G 1M8, Canada (e-mail: cysu@ alcor. concordia.ca).

Color versions of one or more of the figures in this paper are available online at http://ieeexplore.ieee.org.

Digital Object Identifier 10.1109/TAC.2010.2053737 